\title{
Effectiveness of combining feedback about lung age or exhaled CO levels with very brief advice (VBA) and support for smoking cessation in primary care compared to giving VBA and support alone (the LENKA study)
}

Statistical Analysis Plan

Version Number: 1.0

Date: $9^{\text {th }}$ April 2020

Prepared by: Alice Sitch

Reviewed and approved by: Amanda Farley and Rachel Jordan 


\section{Introduction}

This document gives a statistical analysis plan for the LENKA study, and should be read in conjunction with the current study protocol.

\section{Study Design}

The LENKA study is a three-arm, non-blinded parallel individually randomised study which will be conducted in primary care facilities in the Republic of North Macedonia. Outcomes will evaluate the differences between the interventions and control in the proportion who quit smoking (confirmed and self-reported), attempts to quit smoking, changes in motivation to quit and reduction in smoking at four weeks, 12 weeks and 26 weeks. The study will also contain a process evaluation and a cost-effectiveness analysis.

\subsection{Setting}

Eligible participants will be recruited from approximately 30 primary care practices in the Republic of North Macedonia. Smokers who are attending primary care practices, for a consultation with the GP about themselves, for any reason and fit the eligibility criteria, regardless of their motivation to quit smoking, will be invited to take part in the research study and given a patient information leaflet before giving informed consent to participate in the trial. Screening data will be collected from each GP practice; GPs will provide anonymised basic information (age, sex, number of cigarettes smoked per day) for those who meet study eligibility criteria but choose not to take part in the study, to the study team. This data is routinely collected in the GP practices. The research team will summarise this information (mean age; \% males vs. $\%$ females; mean number of cigarettes smoked per day) at the end of the trial, and compare this with summarised information among those who took part in this study. This will determine how representative the included study population are when compared to the general population. 


\subsection{Eligibility criteria}

Inclusion criteria:

- Current smoker, smoking at least 10 cigarettes (manufactured or roll up) per day

- Age $35+$ years

Exclusion criteria:

- Deemed unsuitable to participate in the trial by GP: e.g. cognitive impairment, recent bereavement, terminal illness

- Standard contraindications for spirometry (see protocol appendix 2)

- Previously received VBA to quit smoking

- Currently using e-cigarettes or NRT

- Currently attempting to quit smoking

\subsection{Intervention}

\subsubsection{Trial arm 1: Lung age with very brief advice and support to quit smoking $(V B A+L A)$}

In the lung age intervention arm (VBA+LA), GPs will explain to patients that smoking can cause accelerated lung function decline and that this can be understood in terms of "lung age". GPs will also explain that smoking cessation slows down the rate of lung function decline, although it cannot repair the damage that is already done. GPs will assess lung age using a hand held spirometer (Vitalograph COPD-6). This is a simple device which needs little training to administer and provides a measure of FEV1 and FEV6 as a proxy for FVC. Three blows are undertaken without the use of bronchodilators. Lung age is displayed by the Vitalograph COPD6 machine, and an explanation of what that means will be fed back to the patient by the GP using the best blow. The best blow will be determined by the blow associated with the lowest lung age reading. If lung age is equal to or less than the individual's chronological age, they will be informed that the test result is normal. However, they will be advised that it is still important to stop smoking before the damage is done. If lung age is greater than chronological age, they will be given the "lung age" in years and be advised that although they can't reverse the damage 
quitting smoking will prevent further damage. This will be combined with very brief advice for smoking cessation to all, with support to quit smoking for those who choose to attempt quitting (as described in the control arm).

\subsubsection{Trial arm 2: Exhaled CO with very brief advice and support to quit smoking $(\mathrm{VBA}+\mathrm{CO})$}

In the exhaled $\mathrm{CO}$ intervention arm $(\mathrm{VBA}+\mathrm{CO})$, GPs will explain to patients that cigarette smoke contains $\mathrm{CO}$, and that at high concentrations this is toxic. $\mathrm{CO}$ displaces oxygen in the blood, compromising the transport of oxygen around the body and increasing cardiovascular risk. GPs will assess exhaled CO using a Bedfont piCO Smokerlyzer. This is a simple device that needs little training to administer and gives parts per million (ppm) of CO in exhaled breath. Participants are required to hold their breath for 15 seconds and then blow out into the device, which then gives a reading. The $\mathrm{CO}$ level and an explanation will be fed back to the patient by the GP. This will be combined with very brief advice for smoking cessation for all, with support to quit smoking for those choosing to attempt quitting (as described in the control arm). For participants wishing to quit and undertaking the support to quit, in this arm exhaled $\mathrm{CO}$ will also be repeated and fed-back to the participant at each support session.

\subsection{Comparator}

\subsubsection{Trial arm 3: Very brief advice alone and support to quit smoking (VBA only)}

GPs will deliver VBA. This includes “Asking” a patient's smoking status, "Advising” current smokers to quit smoking and "Acting" to set up appropriate support to quit for patients who are willing to make a quit attempt. Within the context of the trial, participants will have already been asked if they are a current smoker in order to assess eligibility, and therefore GPs will:

- Confirm smoking status

- Advise 
$\circ$ about the harms of smoking

$\circ$ benefits of quitting

o to stop smoking and the best way to stop

- Act

○ For participants who do not want quit smoking (i.e. not at all, or not yet), the GP will advise that the offer of support will be open should they want to take it up another time.

- For participants who want to quit smoking, the GP will set a plan for quitting with the patient including quit date, advise on how to deal with cravings/withdrawal and a plan for support (pharmacological and behavioural).

- For all participants (regardless of quit intention) give smoking information leaflet

\subsection{Randomisation}

\subsubsection{Sequence generation}

The randomisation sequence will be computer generated by a statistician, and will be stratified by GP practice.

\subsubsection{Allocation concealment mechanism}

The randomisation sequence will be concealed from recruiters (GPs) until they create a new patient record within the RedCAP database after baseline questionnaire completion at the point of randomisation. 


\subsection{Blinding}

Given the nature of the intervention and control condition, once participants are allocated to a trial arm the allocation will be known by the GP and the patient. The outcome questionnaires and cotinine saliva test will be self-administered and blind from the GP providing the intervention, where possible.

\subsection{Sample size}

To detect a difference of 5\% in the primary outcome between the control and each intervention arm at 4 weeks (3\% VBA vs $8 \%$ VBA+LA vs $8 \% \mathrm{VBA}+\mathrm{CO})$ with $90 \%$ power and a significance level of $2.5 \%$, we would need 1182 participants in total (394 participants per arm).

\subsection{Outcomes}

\subsubsection{Primary effectiveness outcome}

- Quitting smoking at 4 weeks (7-day point prevalence self-reported abstinence, confirmed with salivary cotinine level of: (1) <10ng/ml, or (2) <100ng/ml for those who report exposure to second hand cigarette smoke in the home on a daily basis, or (3) $\geq 10 \mathrm{ng} / \mathrm{ml}$ in those who report using nicotine replacement therapy/e-cigarettes at any timepoint, irrespective of exposure to second hand cigarette smoke)

\subsubsection{Secondary effectiveness outcomes}

- Attempting to quit smoking, measured at 4, 12 and 26 weeks

- Quitting smoking at $4,12 \& 26$ weeks (self report only).

- Quitting smoking at 12 \& 26 weeks (7-day point prevalence, self reported abstinence, confirmed with salivary cotinine level of: (1) $[<10 \mathrm{ng} / \mathrm{ml}]$, or (2) $<100 \mathrm{ng} / \mathrm{ml}$ for those who report exposure to second hand cigarette smoke in the home on a daily basis, or (3) $\geq 10 \mathrm{ng} / \mathrm{ml}$ 
in those who report using Nicotine Replacement Therapy/e-cigarettes at any time point during the study, irrespective of exposure to second hand cigarette smoke).

- Reduction in the number of cigarettes smoked per day at 4, 12 and 26 weeks

- Motivation to quit at $4,12,26$ weeks

\subsubsection{Exploratory outcomes:}

- In a sub-sample, proportion who have quit smoking at 4, 12 and 26 weeks confirmed with $\mathrm{CO}$ monitor tests (7-day point prevalence self-reported abstinence with an exhaled CO reading of $>10 \mathrm{ppm})$. The results will be concealed from participants unless a reading has already been taken earlier that day.

- In a sub-sample, proportion who are quit at 4,12 and 26 weeks (7-day point prevalence self-reported abstinence, confirmed with salivary cotinine level of: (1) $<10 \mathrm{ng} / \mathrm{ml}$, or (2) $<100 \mathrm{ng} / \mathrm{ml}$ for those who report exposure to second hand cigarette smoke indoors in the last 4 days, or (3) $\geq 10 \mathrm{ng} / \mathrm{ml}$ in those who report using nicotine replacement therapy/ecigarettes in the last 4 days, irrespective of exposure to second hand cigarette smoke).

\section{General Considerations}

\subsection{Levels of confidence and p-values}

For the primary analysis estimates will be presented with $95 \%$ and $97.5 \%$ confidence intervals. P-values will be 2 -tailed, and a p-value of $<0.025$ will be considered statistically significant. For all other analyses, estimates will be displayed with $95 \%$ confidence intervals and a p-value of $<0.05$ will be considered statistically significant.

\subsection{Protocol violations and exclusions from the study}

Analyses will be performed on consenting patients. 


\subsection{Missing data}

Primary and secondary analyses of the proportion who quit smoking will include all patients randomised into the study. Following the Russell Standard for the reporting of smoking cessation outcomes in trials, (1) those who are lost to follow up will remain in the analysis and will be counted as smokers. Patients who have died or are untraceable will be excluded from the analysis. For other outcomes, we will use a complete case analysis.

During the course of the study, one of the recruiting GPs died. All patients recruited at this practice received the intervention and were contacted for the 4 week and 12 week follow up. However, no patients were contacted for the 26 week follow up. Data collected up until and including the 12 week follow up from patients recruited at this practice will be included in the analysis, but these patients will be excluded from the analysis of 26 week follow up data.

\subsection{Interim analyses}

If requested by the TSC for monitoring, interim analyses will be undertaken by the trial

statistician and communicated back to the Trial Steering Committee (TSC) only with the study team blinded to these results.

Sub-analyses of the collected data may be performed for small projects such as student projects. This will not prejudice the main analysis of the trial; analyses which includes comparisons of trial arm will only be performed after the main trial analysis.

\subsection{First main analysis for dissemination}

The first set of analyses of all study data for public dissemination will be started once the last patient entered into the study has completed follow up. 


\section{Proposed Analyses}

\subsection{Primary analysis}

For the primary analysis we will calculate relative risk, and corresponding confidence intervals, comparing each of the two intervention groups with the control group using a Poisson model with robust standard errors adjusting for centre with a random effect.

\subsection{Secondary analysis}

We will report the baseline characteristics of the participants and the outcomes at baseline and follow-up by randomization group, using appropriate summaries.

For the secondary outcomes we will calculate relative risk, and corresponding confidence intervals, comparing each of the two intervention groups with the control group using a Poisson model with robust standard errors adjusting for centre with a random effect.

Additionally, for all outcomes the number of participants achieving the outcome and the number of participants evaluated for the outcome will be displayed by group with unadjusted relative risks comparing the group and $95 \%$ confidence intervals.

We will look to validate the outcome of confirmed quitting (as used in the primary outcome) by comparing this outcome to the exploratory outcomes (see 2.8.3) in a subset of participants at the three time points. We will produce $2 \times 2$ tables to demonstrate the association between these outcomes, and provide estimates comparing the methods with $95 \%$ confidence intervals.

\subsection{Planned subgroup analyses}

We will investigate the potential of differing treatment effect in the primary outcome across the subgroups: 
- $\quad \operatorname{sex}(\mathrm{male} / \mathrm{female})$

- binary categorization of motivation to quit

- Comorbidity (present/not present)

\subsection{Sensitivity analyses}

We will consider undertaking a sensitivity analysis including only those who received the behavioural support following the VBA.

\subsection{Additional analyses}

Analyses will also look to quantify directly the difference in the outcomes between the two intervention groups.

Across all interventions and for each intervention separately, we will look at the difference in smoking status over time. This analysis will account for the paired nature of the data, with McNemar's test used for simple analyses, and multilevel logistic regression used for more complex analyses.

We will investigate whether participant characteristics are associated with confirmed quitting and motivation at the three time points with adjustment for trial arm. We will use a multilevel logistic model to perform this analysis.

We will describe the change in motivation before and after the delivery of the intervention, and investigate if becoming motivated to quit is associated with quitting smoking for the whole group and by trial arm. We will use a Poisson regression model with robust standard error estimates for this analysis.

We will also investigate the following process outcomes, using descriptive analysis methods to summarise the: 
- Proportion of participants who set a quit date, and average length of time between randomisation and quitting

- Average number of behavioural support visits in participants who set a quit date

- Use of pharmacological support to quit in those attempting to quit

- Proportion of participants who received VBA components

- Proportion of participants who received biofeedback (LA, CO reading and explanation) appropriate to trial arm

- Average number of times $\mathrm{CO}$ is repeated in VBA-CO arm

- Fidelity to VBA/CO/LA intervention delivery in a sample of recorded consultations

\subsection{Qualitative and Health Economic analyses}

Qualitative and health economic analyses will be fully specified in the protocol and in separate plans.

Any deviations from this plan will be described in the final report.

\subsection{References}

(1) West R, Hajek P, Stead L, Stapleton J (2005) Outcome criteria in smoking cessation trials: proposal for a common standard Addiction 100 (3): 299-303 